\title{
Positive solutions of fractional differential equations involving the Riemann-Stieltjes integral boundary condition
}

\author{
Qilin Song ${ }^{1}$ and Zhanbing Bai ${ }^{*}$ (B)
}

\section{"Correspondence: \\ zhanbingbai@163.com \\ ${ }^{1}$ College of Mathematics and \\ System Science, Shandong \\ University of Science and \\ Technology, Qingdao, P.R. China}

\begin{abstract}
In this article, the following boundary value problem of fractional differential equation with Riemann-Stieltjes integral boundary condition

$$
\left\{\begin{array}{lc}
D_{0+}^{\alpha} u(t)+\lambda f(t, u(t), u(t))=0, & 0<t<1, n-1<\alpha \leq n \\
u^{(k)}(0)=0, \quad 0 \leq k \leq n-2, & u(1)=\int_{0}^{1} u(s) d A(s)
\end{array}\right.
$$

is studied, where $n-1<\alpha \leq n, \lambda>0, D_{0+}^{\alpha}$ is the Riemann-Liouville fractional derivative, $A$ is a function of bounded variation, $\int_{0}^{1} u(s) d A(s)$ denotes the Riemann-Stieltjes integral of $u$ with respect to $A$. By the use of fixed point theorem and the properties of mixed monotone operator theory, the existence and uniqueness of positive solutions for the problem are acquired. Some examples are presented to illustrate the main result.
\end{abstract}

MSC: $34 \mathrm{~A} 08 ; 34 \mathrm{~B} 18 ; 35 J 05$

Keywords: Riemann-Stieltjes integral; Mixed monotone operator; Fixed point theorem; Existence and uniqueness

\section{Introduction}

Differential equations of fractional order occur frequently in many different research areas and engineering, such as chemistry, optics, thermal systems, signal processing, system identification, etc. Many researchers obtained the existence results of solution of fractional differential equation with initial value problem or boundary value problem [1-23]. In [2], by means of a fixed point theorem, Bai et al. obtained the existence and multiplicity of positive solutions for the singular fractional boundary value problem

$$
\left\{\begin{array}{l}
D_{0+}^{\alpha} u(t)+f\left(t, u(t), D_{0+}^{v} u(t), D_{0+}^{\mu} u(t)\right)=0, \\
u(0)=u^{\prime}(0)=u^{\prime \prime}(0)=u^{\prime \prime}(1)=0
\end{array}\right.
$$

where $3<\alpha \leq 4,0<v \leq 1,1<\mu \leq 2, D_{0+}^{\alpha}$ is the Riemann-Liouville fractional derivative, $f$ is a Carathédory function, and $f(t, x, y, z)$ is singular at the value 0 of its arguments $x, y, z$. Some recent contributions of fractional differential equations can be seen in $[1-8,10-23]$.

(c) The Author(s) 2018. This article is distributed under the terms of the Creative Commons Attribution 4.0 International License (http://creativecommons.org/licenses/by/4.0/), which permits unrestricted use, distribution, and reproduction in any medium, provided you give appropriate credit to the original author(s) and the source, provide a link to the Creative Commons license, and indicate if changes were made. 
Recently, the theory of integral boundary value problems has become a new area of investigation (see $[5,8,20])$. Cabada and Wang [5] have considered the following nonlinear fractional differential equations with integral boundary conditions for the first time:

$$
\left\{\begin{array}{l}
{ }^{C} D_{0+}^{\alpha} u(t)+f(t, u(t))=0, \quad 0<t<1, \\
u(0)=u^{\prime \prime}(0)=0, \quad u(1)=\lambda \int_{0}^{1} u(s) d s,
\end{array}\right.
$$

where $2<\alpha<3,0<\lambda<2,{ }^{C} D_{0+}^{\alpha} u(t)$ is the Caputo fractional derivative, and $f:[0,1] \times$ $[0, \infty) \rightarrow[0, \infty)$ is a continuous function. They used Guo-Krasnoselskii's fixed point theorem to get the existence of positive solutions.

Zhang [20] has actually studied the boundary value problem with the boundary condition involving parameter $\lambda$

$$
\left\{\begin{array}{l}
D_{0+}^{\alpha} u(t)+f(t, u(t))=0, \quad t \in(0,1), \\
u(0)=u^{\prime}(0)=0, \quad D_{0+}^{\beta} u(1)=\lambda \int_{0}^{1} D_{0+}^{\beta} u(t) d A(t),
\end{array}\right.
$$

where $2<\alpha \leq 3,0<\beta \leq 1$ are real numbers and $\int_{0}^{1} D_{0+}^{\beta} u(t) d A(t)$ denotes a RiemannStieltjes integral. By means of the monotone iterative technique and the inequalities associated with Green's function, they obtained the existence of nontrivial solutions or positive solutions.

In [8], Feng and Zhai used a new fixed point theorem [19] to consider the following problem:

$$
\left\{\begin{array}{l}
D_{0+}^{\alpha} u(t)+\lambda f(t, u(t), u(t))=0, \quad t \in(0,1), \\
u(0)=u^{\prime}(0)=0, \quad u(1)=\int_{0}^{1} q(t) u(t) d t,
\end{array}\right.
$$

where $2<\alpha \leq 3,0<\beta \leq 1$ are real numbers, $f:[0,1] \times[0, \infty) \times[0, \infty) \rightarrow[0, \infty)$ is a continuous function, $q:[0,1] \rightarrow[0, \infty), q \in L^{1}[0,1], \sigma_{1}=\int_{0}^{1} s^{\alpha-1}(1-s) q(s) d s>0, \sigma_{2}=$ $\int_{0}^{1} s^{\alpha-1} q(s) d s<1$. They obtained the existence and uniqueness of positive solutions.

Motivated by the mentioned excellent works, in this paper, we consider the following problem:

$$
\begin{array}{ll}
D_{0+}^{\alpha} u(t)+\lambda f(t, u(t), u(t))=0, & 0<t<1, n-1<\alpha \leq n, \\
u^{(k)}(0)=0, \quad 0 \leq k \leq n-2, & u(1)=\int_{0}^{1} u(s) d A(s),
\end{array}
$$

where $D_{0_{+}}^{\alpha}$ is the Riemann-Liouville fractional derivative, $\lambda>0, A$ is a function of bounded variation, $\int_{0}^{1} u(s) d A(s)$ denotes the Riemann-Stieltjes integral of $u$ with respect to $A$. The problem studied in [8] is a special case of our paper for $1<\alpha \leq 2$ and $A(s)$ is differentiable such that $\int_{0}^{1} s^{\alpha-1}(1-s) q(s) d s>0$ and $\int_{0}^{1} s^{\alpha-1} q(s) d s<1$. In the current paper, $A$ is a function of bounded variation such that $\int_{0}^{1} G(t, s) d A(t) \geq 0$ and $\int_{0}^{1} t^{\alpha-1} d A(t)<1$ for $s \in[0,1]$, where $G(t, s)$ will be defined in the next section.

The rest of this paper is organized as follows. In Sect. 2, we recall some definitions, theorems, and lemmas. In Sect. 3, we investigate the existence and uniqueness of positive solution for problem (1.1), (1.2). In Sect. 4, we present some examples to illustrate our main results. 


\section{Preliminaries and lemmas}

Suppose that $(E,\|\cdot\|)$ is a real Banach space, $P \subset E$ is a normal cone. For all $x, y \in E$, the notation $x \sim y$ means that there exist $\lambda, \mu>0$ such that $\lambda x \leq y \leq \mu x$. Clearly, $\sim$ is an equivalence relation. Given $h>\theta$ (i.e., $h \geq \theta, h \neq \theta$ ), we denote $P_{h}=\{x \in E \mid x \sim h\}$. It is easy to see that $P_{h} \subset P$ is convex and $\lambda P_{h}=P_{h}$ for all $\lambda>0$. We refer the readers to the references [9] and [19] for details.

Definition 2.1 ([19]) $T: P \times P \rightarrow P$ is said to be a mixed monotone operator if $T(x, y)$ is increasing in $x$ and decreasing in $y$, i.e., $u_{i}, v_{i}(i=1,2) \in P, u_{1} \leq u_{2}, v_{1} \geq v_{2}$ imply $T\left(u_{1}, v_{1}\right) \leq$ $T\left(u_{2}, v_{2}\right)$. The element $x \in P$ is called a fixed point of $T$ if $T(x, x)=x$.

Theorem 2.1 ([9]) Suppose that $P$ is a normal cone of $E, T: P \times P \rightarrow P$ is a mixed monotone operator such that the following conditions hold:

(A1) There exists $h \in P$ with $h \neq \theta$ such that $T(h, h) \in P_{h}$.

(A2) For any $u, v \in P$ and $t \in(0,1)$, there exists $\varphi(t) \in(t, 1]$ such that $T\left(t u, t^{-1} v\right) \geq$ $\varphi(t) T(u, v)$.

Then operator $T$ has a unique fixed point $x^{*}$ in $P_{h}$. Moreover, for any initial $x_{0}, y_{0} \in P_{h}$ constructing successively the sequences

$$
x_{n}=T\left(x_{n-1}, y_{n-1}\right), \quad y_{n}=T\left(y_{n-1}, x_{n-1}\right), \quad n=1,2, \ldots
$$

there are $\left\|x_{n}-x^{*}\right\| \rightarrow 0$ and $\left\|y_{n}-x^{*}\right\| \rightarrow 0$ as $n \rightarrow \infty$.

Lemma 2.1 ([22]) The Green's function for the nonlocal boundary value problem (1.1), (1.2) is given by

$$
H(t, s)=\frac{t^{\alpha-1}}{1-\delta} G_{A}(s)+G(t, s)
$$

where

$$
\begin{aligned}
& G(t, s)=\frac{1}{\Gamma(\alpha)} \begin{cases}{[t(1-s)]^{\alpha-1}-(t-s)^{\alpha-1},} & \text { for } 0 \leq s \leq t \leq 1 \\
{[t(1-s)]^{\alpha-1},} & \text { for } 0 \leq t \leq s \leq 1,\end{cases} \\
& G_{A}(s)=\int_{0}^{1} G(t, s) d A(t), \quad \delta=\int_{0}^{1} t^{\alpha-1} d A(t) \neq 1 .
\end{aligned}
$$

Lemma 2.2 ([22]) Let $\delta<1, G_{A}(s) \geq 0$ for $s \in[0,1]$, then the Green's function defined by

(2.1) satisfies:

(1) $H(t, s)>0$ for all $t, s \in(0,1)$;

(2) The following relation holds:

$$
c t^{\alpha-1} G_{A}(s) \leq H(t, s) \leq d t^{\alpha-1} \leq d, \quad t, s \in[0,1],
$$

where the constants $c=\frac{1}{1-\delta}, d=\frac{\left\|G_{A}(s)\right\|}{1-\delta}+\frac{1}{\Gamma(\alpha-1)}$. 


\section{Main results}

Let $E=C[0,1]$ equipped with the maximum norm $\|u\|=\max _{0 \leq t \leq 1}|u(t)|$, then $E$ is a Banach space. Define $P=\{x \in C[0,1] \mid x(t) \geq 0, t \in[0,1]\}$, then $P$ is a normal cone, and for $x, y \in C[0,1]$,

$$
x \leq y \quad \Leftrightarrow \quad x(t) \leq y(t), \quad t \in(0,1) .
$$

\section{Theorem 3.1 Assume that}

(H1) $A$ is a function of bounded variation such that

$$
\int_{0}^{1} G(t, s) d A(t) \geq 0 \text { and } \int_{0}^{1} t^{\alpha-1} d A(t)<1
$$

for $s \in[0,1]$

(H2) $f \in C([0,1] \times[0,+\infty) \times[0,+\infty),[0,+\infty)), f(t, x, y)$ is nondecreasing in $x$ for each $t \in[0,1], y \in[0,+\infty)$ and nonincreasing in $y$ for each $t \in[0,1], x \in[0,+\infty)$;

(H3) $f(t, 0,1) \neq 0, t \in[0,1]$;

(H4) for any $\gamma \in(0,1)$, there exists a constant $\varphi(\gamma) \in(\gamma, 1]$ such that $f\left(t, \gamma x, \gamma^{-1} y\right) \geq$ $\varphi(\gamma) f(t, x, y)$ for any $x, y \in[0,+\infty)$.

Then, for any $\lambda>0$, boundary value problem (1.1), (1.2) has a unique positive solution $u_{\lambda}^{*} \in P_{h}$, where $h(t)=t^{\alpha-1}, t \in[0,1]$. Moreover, for any $u_{0}, v_{0} \in P_{h}$, let

$$
\begin{cases}u_{n+1}=\lambda \int_{0}^{1} H(t, s) f\left(s, u_{n}(s), v_{n}(s)\right) d s, & n=0,1,2, \ldots \\ v_{n+1}=\lambda \int_{0}^{1} H(t, s) f\left(s, v_{n}(s), u_{n}(s)\right) d s, & n=0,1,2, \ldots\end{cases}
$$

there is

$$
u_{n}(t) \rightarrow u_{\lambda}^{*}(t), \quad v_{n}(t) \rightarrow u_{\lambda}^{*}(t) \quad(n \rightarrow \infty)
$$

where $H(t, s)$ is given in Lemma 2.1.

Proof It is well known that $u$ is a solution of the boundary value problem (1.1), (1.2) if and only if

$$
u(t)=\lambda \int_{0}^{1} H(t, s) f(s, u(s), u(s)) d s
$$

where $H(t, s)$ is given in Lemma 2.1. For any $u, v \in P$, define

$$
T_{\lambda}(u, v)(t)=\lambda \int_{0}^{1} H(t, s) f(s, u(s), v(s)) d s .
$$

From (H1), (H2), and (2.2), for any $u_{i}, v_{i} \in P, i=1,2$, such that $u_{1} \geq u_{2}, v_{1} \leq v_{2}$, there is

$$
\begin{aligned}
T_{\lambda}\left(u_{1}, v_{1}\right)(t) & =\lambda \int_{0}^{1} H(t, s) f\left(s, u_{1}(s), v_{1}(s)\right) d s \\
& \geq \lambda \int_{0}^{1} H(t, s) f\left(s, u_{2}(s), v_{2}(s)\right) d s=T_{\lambda}\left(u_{2}, v_{2}\right)(t) .
\end{aligned}
$$

That is to say, $T_{\lambda}: P \times P \rightarrow P$ is a mixed monotone operator. 
Next, we will prove that $T_{\lambda}$ satisfies the conditions of Theorem 2.1. From $(H 4)$, for any $u, v \in P$ and $\gamma \in(0,1)$, we can obtain

$$
\begin{aligned}
T_{\lambda}\left(\gamma u, \gamma^{-1} v\right)(t) & =\lambda \int_{0}^{1} H(t, s) f\left(s, \gamma u(s), \gamma^{-1} v(s)\right) d s \\
& \geq \lambda \int_{0}^{1} H(t, s) \varphi(\gamma) f(s, u(s), v(s)) d s \\
& =\varphi(\gamma) T_{\lambda}(u, v)(t), \quad t \in[0,1] .
\end{aligned}
$$

That is, $T_{\lambda}\left(\gamma u, \gamma^{-1} v\right) \geq \varphi(\gamma) T_{\lambda}(u, v)$ for any $u, v \in P$ and $\gamma \in(0,1)$. So condition $(A 2)$ in Theorem 2.1 is satisfied. Then, from $(H 3),(H 4)$, and Lemma 2.2, we get

$$
\begin{aligned}
T_{\lambda}(h, h)(t) & =\lambda \int_{0}^{1} H(t, s) f(s, h(s), h(s)) d s \\
& \geq \lambda c t^{\alpha-1} \int_{0}^{1} G_{A}(s) f(s, 0,1) d s .
\end{aligned}
$$

On the other hand,

$$
\begin{aligned}
T_{\lambda}(h, h)(t) & =\lambda \int_{0}^{1} H(t, s) f(s, h(s), h(s)) d s \\
& \leq \lambda d t^{\alpha-1} \int_{0}^{1} f(s, 0,1) d s .
\end{aligned}
$$

Let $r_{1}=c \int_{0}^{1} G_{A}(s) f(s, 0,1) d s$, and $r_{2}=d \int_{0}^{1} f(s, 0,1) d s$, then

$$
\lambda r_{1} t^{\alpha-1} \leq T_{\lambda}(h, h) \leq \lambda r_{2} t^{\alpha-1}
$$

Obviously, $r_{1}, r_{2}>0$, so $T_{\lambda}(h, h) \in P_{h}$, condition $(A 1)$ in Theorem 2.1 is satisfied. Then, from Theorem 2.1, there exists a unique $u_{\lambda}^{*} \in P_{h}$ such that $T_{\lambda}\left(u_{\lambda}^{*}, u_{\lambda}^{*}\right)=u_{\lambda}^{*}$. We can check that $u_{\lambda}^{*}$ is the unique positive solution of problem (1.1), (1.2). For any initial value $u_{0}, v_{0} \in P_{t^{\alpha-1}}$, establish the sequence $u_{n+1}=T_{\lambda}\left(u_{n}, v_{n}\right), v_{n+1}=T_{\lambda}\left(v_{n}, u_{n}\right), n=0,1,2, \ldots$, one has $u_{n} \rightarrow u_{\lambda}^{*}, v_{n} \rightarrow u_{\lambda}^{*}(n \rightarrow \infty)$, i.e.,

$$
\begin{cases}u_{n+1}(t)=\lambda \int_{0}^{1} H(t, s) f\left(s, u_{n}(s), v_{n}(s)\right) d s \rightarrow u_{\lambda}^{*}(t), & n \rightarrow \infty \\ v_{n+1}(t)=\lambda \int_{0}^{1} H(t, s) f\left(s, v_{n}(s), u_{n}(s)\right) d s \rightarrow u_{\lambda}^{*}(t), & n \rightarrow \infty\end{cases}
$$

The proof is complete.

\section{Examples}

Example 4.1 Consider the following boundary value problem:

$$
\begin{aligned}
& D_{0+}^{\alpha} u(t)+\lambda\left[u(t)^{a}+(u(t)+c)^{b}\right]=0, \quad 0<t<1 ; \\
& u^{(k)}(0)=0, \quad 0 \leq k \leq n-2, \quad u(1)=\int_{0}^{1} u(s) d A(s),
\end{aligned}
$$


where $\alpha=2.5, a=\frac{1}{2}, b=-\frac{1}{2}, c=1$, and $A(t)=\frac{1}{3} e^{t}$. Obviously, $\delta=\int_{0}^{1} t^{\alpha-1} d A(t)<1$. Clearly, $f(t, x, y)=x^{a}+(y+c)^{b}$ is increasing in $x$ for any $y \geq 0$, and decreasing in $y$ for any $x \geq 0$, $f(t, 0,1)=\sqrt{2} / 2 \neq 0$. Moreover,

$$
\begin{aligned}
f\left(t, \gamma x, \gamma^{-1} y\right) & \geq \gamma^{a} x^{a}+\gamma^{-b}(y+c)^{b} \\
& \geq \gamma^{\max \{a,|b|\}}\left(x^{a}+(y+c)^{b}\right) \\
& =\varphi(\gamma)\left(x^{a}+(y+c)^{b}\right) \\
& =\varphi(\gamma) f(t, x, y) .
\end{aligned}
$$

So, $\varphi(\gamma)=\sqrt{\gamma}>\gamma$ for $\gamma \in(0,1)$, the conditions of Theorem 3.1 are all satisfied. Then problem (4.1), (4.2) has a unique solution $u_{\lambda}^{*} \in P_{t^{\alpha-1}}$. For any initial value $u_{0}, v_{0} \in P_{t^{\alpha-1}}$, we can set the following sequence:

$$
\begin{cases}u_{n+1}=\lambda \int_{0}^{1} H(t, s)\left[u(t)^{a}+(u(t)+c)^{b}\right] d s, & n=0,1,2, \ldots \\ v_{n+1}=\lambda \int_{0}^{1} H(t, s)\left[u(t)^{a}+(u(t)+c)^{b}\right] d s, & n=0,1,2, \ldots\end{cases}
$$

then we have

$$
u_{n}(t) \rightarrow u_{\lambda}^{*}(t), \quad v_{n}(t) \rightarrow u_{\lambda}^{*}(t) \quad(n \rightarrow \infty)
$$

where $H(t, s)$ is given in Lemma 2.1 .

Example 4.2 For problem (4.1), (4.2), let $\alpha=2.5, a=\frac{1}{4}, b=-\frac{1}{5}, c=1$, and

$$
A(t)= \begin{cases}\sin \alpha \pi t+1, & 0<t<\frac{1}{\alpha} \\ -\cos \alpha \pi t, & \frac{1}{\alpha} \leq t<1\end{cases}
$$

It is easy to check that $\delta=\int_{0}^{1} t^{\alpha-1} d A(t)<1$. Clearly, $f(t, x, y)$ is increasing in $x$ for any $t \in[0,1], y \geq 0$, and decreasing in $y$ for any $t \in[0,1], x \geq 0, f(t, 0,1)=2^{-\frac{1}{5}} \neq 0$. Moreover,

$$
\begin{aligned}
f\left(t, \gamma x, \gamma^{-1} y\right) & \geq \gamma^{\frac{1}{4}} x^{\frac{1}{4}}+\gamma^{\frac{1}{5}}(y+c)^{-\frac{1}{5}} \\
& \geq \gamma^{\frac{1}{4}}\left(x^{\frac{1}{4}}+(y+c)^{-\frac{1}{5}}\right) \\
& =\varphi(\gamma)\left(x^{\frac{1}{4}}+(y+c)^{-\frac{1}{5}}\right) \\
& =\varphi(\gamma) f(t, x, y)
\end{aligned}
$$

where $\varphi(\gamma)=\gamma^{\frac{1}{4}}>\gamma$, the conditions of Theorem 3.1 all hold. So this problem has a unique positive solution $u_{\lambda}^{*} \in P_{t^{\alpha-1}}$.

\section{Conclusion}

The research of fractional calculus and integral boundary value conditions has become a new area of investigation. By the use of fixed point theorem and the properties of mixed monotone operator theory, the existence and uniqueness of positive solutions for the 
problem are acquired. Two examples are presented to illustrate the main results. The conclusion obtained in this paper will be very useful in the application point of view. Also, we expect to find some applications in more nonlinear problems.

\section{Acknowledgements}

Not applicable.

\section{Funding}

This work is supported by NSFC (11571207), the Taishan Scholar project and SDUST graduate innovation project SDKDYC170343.

\section{Competing interests}

The authors declare that they have no competing interests.

\section{Authors' contributions}

All authors contributed equally to the writing of this paper. All authors read and approved the final manuscript.

\section{Publisher's Note}

Springer Nature remains neutral with regard to jurisdictional claims in published maps and institutional affiliations.

Received: 18 February 2018 Accepted: 5 May 2018 Published online: 16 May 2018

\section{References}

1. Bai, Z., Dong, X., Yin, C.: Existence results for impulsive nonlinear fractional differential equation with mixed boundary conditions. Bound. Value Probl. 2016, Article ID 63 (2016). https://doi.org/10.1186/s13661-016-0573-z

2. Bai, Z., Sun, W.: Existence and multiplicity of positive solutions for singular fractional boundary value problems. Comput. Math. Appl. 63, 1369-1381 (2012)

3. Bai, Z., Zhang, S., Sun, S., Yin, C.: Monotone iterative method for fractional differential equations. Electron. J. Differ. Equ. 2016, Article ID 6 (2016)

4. Bai, Z., Zhang, Y.: Solvability of fractional three-point boundary value problems with nonlinear growth. Appl. Math. Comput. 218, 1719-1725 (2011)

5. Cabada, A., Wang, G.: Positive solutions of nonlinear fractional differential equations with integral boundary value conditions. Abstr. Appl. Anal. 2012, Article ID 403 (2012)

6. Cui, Y.: Uniqueness of solution for boundary value problems for fractional differential equations. Appl. Math. Lett. 51, 48-54 (2016)

7. Dong, H., Guo, B., Yin, B.: Generalized fractional supertrace identity for Hamiltonian structure of NLS-MKdV hierarchy with self-consistent sources. Anal. Math. Phys. 6, 199-209 (2016)

8. Feng, H., Zhai, C.: Properties of positive solutions to a class of fractional differential equations with parameters and integral boundary value conditions. Appl. Math. Mech. 38(7), 818-826 (2017) (in Chinese). https://doi.org/10.21656/1000-0887.380124

9. Guo, D.: Fixed points of mixed monotone operators with application. Appl. Anal. 34, 215-224 (1988)

10. Ji, D., Bai, Z., Ge, W.: The existence of countably many positive solutions for singular multipoint boundary value problems. Nonlinear Anal., Theory Methods Appl. 72, 955-964 (2010)

11. Li, H., Sun, J.: Positive solutions of superlinear semipositone nonlinear boundary value problems. Comput. Math. Appl. 61, 2806-2815 (2011)

12. LV, W., Wang, F.: Adaptive tracking control for a class of uncertain nonlinear systems with infinite number of actuator failures using neural networks. Adv. Differ. Equ. 2017, Article ID 374 (2017)

13. Song, Q., Dong, X., Bai, Z., Chen, B.: Existence for fractional Dirichlet boundary value problem under barrier strip conditions. J. Nonlinear Sci. Appl. 10, 3592-3598 (2017)

14. Tian, Y., Bai, Z.: Existence results for the three-point impulsive boundary value problem involving fractional differential equations. Comput. Math. Appl. 59, 2601-2609 (2010)

15. Wang, G., Agarwal, R., Cabada, A.: Existence results and monotone iterative technique for systems of nonlinear fractional differential equations. Appl. Math. Lett. 25(6), 1019-1024 (2012)

16. Wang, Z:: A numerical method for delayed fractional-order differential equations. J. Appl. Math. 2013, Article ID $256071(2013)$

17. Wang, Z., Huang, X., Shi, G.: Analysis of nonlinear dynamics and chaos in a fractional order financial system with time delay. Comput. Math. Appl. 62, 1531-1539 (2011)

18. Yuan, C.: Multiple positive solutions for $(n-1,1)$-type semipositone conjugate boundary value problems of nonlinear fractional differential equations. Electron. J. Qual. Theory Differ. Equ. 2010, Article ID 36 (2010)

19. Zhai, C., Zhang, L.: New fixed point theorems for mixed monotone operators and local existence uniqueness of positive solutions for nonlinear boundary value problems. J. Math. Anal. Appl. 382, 594-614 (2011)

20. Zhang, H.: Iterative solutions for fractional nonlocal boundary value problems involving integral conditions. Bound. Value Probl. 2016, Article ID 3 (2016)

21. Zhang, W., Bai, Z., Sun, S.: Extremal solutions for some periodic fractional differential equations. Adv. Differ. Equ. 2016 Article ID 179 (2016)

22. Zhang, X., Han, Y.: Existence and uniqueness of positive solutions for higher order nonlocal fractional differential equations. Appl. Math. Lett. 25, 555-560 (2012)

23. Zou, Y., Liu, L., Cui, Y.: The existence of solutions for four-point coupled boundary value problems of fractional differential equations at resonance. Abstr. Appl. Anal. 2014, Article ID 314083 (2014) 